\title{
Solidification of Electronic Materials: An Overview
}

\author{
André Phillion
}

Electronic materials are now found throughout the world and are used in virtually every aspect of life. Consider a backcountry canoe trip (one of my favorite activities) - although nature surrounds the canoeist, the presence of perhaps a radio and light-emitting diode flashlight, as well as a solar panel for recharging batteries and energy storage is a reminder of modern technology. The radio, flashlight, and solar panel all contain electronic materials to enable component usage, heavily processed to meet purity, reliability, and/or dopant requirements.

Solidification affects three aspects of electronics and electronic materials production. First, an electronic material must meet strict purity requirements to optimize related physical and functional properties. High-purity materials can only be produced through excellent control of the casting process. Second, solidification is used during component assembly to physically join or solder various electrical devices to the underlying printed circuit board. Third, the quality of the solder will affect the long-term in-service reliability of the electronic component.

This issue of JOM explores the topic of electronic materials solidification by examining the fundamentals along with processing-related questions. In the past few years, advances in this field have occurred in the areas of leadfree solders, multicrystalline silicon production for photovoltaic cells, and component assembly processing techniques.

The first two papers examine solidi- fication mechanisms in silicon alloys for solar cell applications. The major challenge in this industry is to meet purity requirements enabling high cell efficiency while reducing costs. In the first paper, L. Arnberg et al. critically assess a number of the most common methods for producing multicrystalline silicon. Although the Czochralski crystal pulling and Bridgman-type directional solidification methods currently dominate the market, new techniques such as electro-magnetic casting and direct ribbon casting are shown to have potential for significantly higher productivity. Next, the use of a crucible cover during directional solidification is examined by B. Gao et al. as a method for improving alloy purity levels. Numerical simulations show that while the use of a crucible can significantly reduce carbon and oxygen levels in the melt, new design strategies are needed to ensure that the crucible cover does not react with the $\mathrm{SiO}$ gas.

The conversion to lead-free solder alloys and increased warpage due to the use of thinner microelectronic packaging technologies has increased the risk of solder joint defects during component assembly. In the third paper, by R.S. Sidhu et al., the common head-and-pillow solder defect that occurs between fine-pitch ball grid array (BGA-type) electronic packages and the motherboard during surface mount assembly is examined and the mechanisms related to various aspects of design and solder quality that affect defect formation are discussed. It is shown that even small variations in solder chemistry can lead to increased risk of defect formation; chemistries that reduce oxide formation or extend solidification undercooling are beneficial as they increase the time available for wetting between the solder ball and paste.

Solidification mechanisms in tinrich lead-free solders is also the focus of the fourth paper, by M. Felberbaum et al. In this work, the mechanisms responsible for the beneficial effects of $\mathrm{Ni}$ in $\mathrm{Sn}-0.7 \mathrm{Cu}$ solder are investigated through the use of Bridgman solidification experiments and microscopy. It is shown that changes in microstructure morphology are due mostly to the segregation of impurity elements, particularly lead, and not the nickel content. Instead, the nickel addition is beneficial as it improves the fluidity and reduces the freezing range.

In summary, solidification issues within electronic materials are diverse, and can provide many avenues for scientific research and development. Novel solidification techniques, especially in multicrystalline silicon for photovoltaics and component reliability, are necessary for improving the economics of renewable-energy sources. The articles in this issue capture only a small subset of current unanswered questions while highlighting key achievements and remaining challenges.

André Phillion is an assistant professor with the Okanagan School of Engineering, The University of British Columbia and is the advisor to JOM from the Solidification Committee of the Materials Processing \& Manufacturing Division of TMS.

\section{André Phillion is a TMS Member!}

To read more about him, turn to page 9. To join TMS, visit www.tms.org/Society/Membership.aspx. 